\title{
Nomogram for Predicting Overall Survival in Patients With Invasive Micropapillary Carcinoma After Breast-Conserving Surgery: A Population- Based Analysis
}

\section{Yuting Zhao}

the First Affiliated Hospital of Xi'an Jiaotong University

Shouyu Li

the First Affiliated Hospital of Xi'an Jiaotong University

\section{Lutong Yan}

the First Affiliated Hospital of Xi'an Jiaotong University

\section{Zejian Yang}

the First Affiliated Hospital of Xi'an Jiaotong University

\section{$\mathrm{Na}$ Chai}

the First Affiliated Hospital of Xi'an Jiaotong University

Pei Qiu

the First Affiliated Hospital of Xi'an Jiaotong University

Jian Zhang

the First Affiliated Hospital of Xi'an Jiaotong University

\section{Huimin Zhang}

the First Affiliated Hospital of Xi'an Jiaotong University

Jianjun He

the First Affiliated Hospital of Xi'an Jiaotong University

Can Zhou ( $\nabla$ zhoucanz2005@126.com)

the First Affiliated Hospital of Xi'an Jiaotong University

\section{Research Article}

Keywords: invasive micropapillary carcinoma, breast-conserving surgery, SEER, overall survival, nomogram, decision curve analyses

Posted Date: December 22nd, 2021

DOI: https://doi.org/10.21203/rs.3.rs-1156745/v1 
License: (c) (i) This work is licensed under a Creative Commons Attribution 4.0 International License. Read Full License 


\section{Abstract}

Background: Due to the rarity of invasive micropapillary carcinoma (IMPC) of the breast, no randomized trial has investigated the prediction of overall survival (OS) for patients with IMPC after breast-conserving surgery (BCS). This study aimed to construct a nomogram for predicting OS in IMPC patients after BCS.

Methods: Using the Surveillance, Epidemiology, and End Results (SEER) database, 481 eligible cases diagnosed with IMPC were collected. OS in IMPC patients after BCS were assessed through multivariable Cox analyses, Harrell's concordance indexes (C-indexes), receiver operating characteristics (ROCs) curves, calibration curves, decision curve analyses (DCA), and survival analyses.

Results: 336 patients were randomly assigned into training cohort and 145 cases in validation cohort. The multivariate Cox regression analyses revealed that age at diagnosis, American Joint Committee on Cancer (AJCC) stage, marital status, hormone receptor status and chemotherapy were significant prognostic factors for OS in conservatively operated IMPC patients. The nomogram had a good prediction performance with the $\mathrm{C}$-indices $0.771(95 \% \mathrm{Cl}, 0.712-0.830)$ and $0.715(95 \% \mathrm{Cl}, 0.603-0.827)$ in training and validation cohorts, respectively, and good consistency between the predicted and observed probability, with calibration curves plotted and the slope was close to 1. Based on calculation of the model, participants in low-risk group had a better OS in comparison with those in high-risk group $(P<$ $0.001)$.

Conclusions: A nomogram was developed to predict individualized risk of OS for IMPC patients after BCS. By risk stratification, this model is expected to guide treatment decision making in improving longterm follow-up strategies for IMPC patients.

\section{Introduction}

Invasive micropapillary carcinoma (IMPC), composed of small, hollow or morula-like clusters of cancer cells and surrounded by clear stromal spaces, is a rare histologic subtype of breast cancer in the World Health Organization (WHO) Classification of Tumors of the Breast in the 2003 and 2012 editions[1-3]. Previous researches have indicated that IMPC tend to show aggressive clinical characteristics, such as significant tendency for lymphovascular invasion (LVI), lymph node metastasis (LNM), and poor prognosis[4-6]. Consequently, IMPC, which has gained increasing attention in comprehensive therapy field of breast cancer, is always over-treated with unnecessary chemotherapy in the past 20 years. One of the main reasons is the limited knowledge on IMPC based on clinical literature researches owing to the rarity of this breast malignancy[6,7]. Moreover, current guidelines are mainly developed on the basis of invasive ductal carcinoma (IDC), underlining the urgent need for more clinical evidence on treatment strategies for IMPC.

As one of the standard treatments of breast cancer, breast-conserving surgery (BCS) followed by radiotherapy has excellent results on overall survival (OS) and relapse-free survival and been widely performed in recent years[8,9]. The efficacy of BCS on IDC rather than IMPC has been extensively 
evaluated. And the precise clinical value of BCS for patients with IMPC is still unknown, due to the lack of large, randomized controlled trials investigated the prognostic prediction of the OS probability for patients with IMPC after BCS. For these reasons, the confirmation of the real-world therapeutic effectiveness on BCS for IMPC patients is urgently needed.

To further explore the therapeutic effect of BCS on IMPC, a large population of female IMPC patients is investigated by using Surveillance, Epidemiology, and End Results (SEER) database. A nomogram is firstly established and validated based on prognostic factors from the Cox multivariate analysis to obtain predicted survival probabilities in IMPC patients underwent BCS. This model is expected to provide guidance on the OS probability for IMPC patients with BCS through important prognosis markers and facilitate the decision-making of follow-up treatment for clinicians.

\section{Materials And Methods}

\subsection{Data source and patient population}

For the current study, data were derived from SEER 18 Regs Research Data with a data user agreement. The SEER ${ }^{*}$ Stat software program (version 8.3.8, http://seer.cancer.gov/seerstat) (Information Management Service, Inc. Calverton, MD, USA) was administrated to select female patients diagnosed with IMPC undergoing BCS from 2000 to 2016 with the inclusion criteria listed as follows: 1) primary tumor site code 8507/3: Ductal carcinoma, micropapillary in the International Classification of Disease for Oncology, third edition (ICD-0-3); 2) Breast-Adjusted American Joint Committee on Cancer (AJCC) 6th stages 0 , I, II or III; 3 ) cancer-directed surgery recode BCS. The exclusion criteria were listed as follows: 1) unknown age at diagnosis, marital status, histopathological differentiation grade, AJCC stage, survival time, estrogen receptor (ER) status or progesterone receptor (PR) status; 2 ) the diagnosis methods of autopsy only or death certificate; 3) AJCC stage IV or grade IV; 4) missing surgical records, or surgery not performed. The screening process was presented in Figure 1. The studied variables included were age at diagnosis, race, years of diagnosis, marital status, histopathological grade, breast-adjusted AJCC 6th TNM stage, ER status, PR status, HER-2 status, radiotherapy, chemotherapy, survival months, vital status, cause of death, type of surgical procedures, and so on. All methods were performed in accordance with the relevant guidelines and regulations. This study was approved by the Ethical Committee of the First Affiliated Hospital of Xi'an Jiaotong University. The SEER data erases the identity information of patients, so there is no need for informed consent from the patients.

\subsection{Data collection and endpoint}

The median follow-up time of the study cohort was 4.92 years (range, 0 month to 15.75 years). The endpoint of the current study was overall survival (OS), which was defined as the time from the date of diagnosis until death from any cause, the date of last follow-up or November 2018.

\subsection{Statistical analysis}


Patients were randomly split to the training cohort and the validation cohort (7:3) by R software rms package. Differences between the cohorts were compared by Pearson's chi-square test or Fisher's exact test when needed. In training cohort, Hazard ratios (HRs) with 95\% confidence intervals (Cls) were calculated through Cox proportional hazards model to estimate prognosis and screen out significant factors to generate nomogram. The nomogram was built in training cohort with the result of Cox multivariate analysis to predict the OS probability using R software rms package. Harrell's concordanceindex (C-index) and receiver operating characteristics (ROC) curves were performed for evaluating the discriminatory ability of the nomogram. Calibration curves were plotted to evaluate the consistency between the predicted and the observed probability. Decision curve analyses (DCA) were performed for clinical usefulness assessment of this predictive model.

Combined with the coefficient of each variable in the Cox regression model, a risk score system was constructed by the following formula[10]:

$\hat{h} i(t)=\hat{h} 0(t) \exp \left(x^{\prime} i \hat{\beta}\right)$

The total cohort was split into high- and low-risk groups with the median risk score as a cut-off value.

Survival curves by risk level and other significant variables for the study patients were calculated using the Kaplan-Meier analysis and compared across groups by log-rank test. Two-sided $P<0.05$ was considered to have statistical significance. Statistical analyses were performed by IBM SPSS version 26.0 and software package $\mathrm{R}$ version 4.0.2.

\section{Result}

\subsection{Patient Characteristics}

A total of 481 participants diagnosed with IMPC were included in the current study. Totally, 336 eligible subjects were randomly assigned to training cohort and 145 subjects were randomly assigned to validation cohort. The clinicopathologic features were shown in Table 1. Among all 481 patients, the mean age was $58.13( \pm 14.17)$ years old, $58.6 \%(282 / 481)$ of them was White race, and $56.5 \%(272 / 481)$ of them were married women. The majority of IMPC cases were moderately and poorly differentiated (grade II and III) $(95.20 \%, 458 / 481)$, AJCC stage III (54.50\%, 262/481), ER positive (85.00\%, 409/481), PR positive $(72.30 \%, 348 / 481)$ and HER-2 negative $(73.90 \%, 212 / 287)$ tumors. In total, $46.8 \%(225 / 481)$ of patients received radiotherapy, and $72.60 \%$ (349/481) had underwent chemotherapy. No significant differences in the clinicopathologic characteristics were observed between training and validation cohorts. 
Table 1

Patient clinical and pathological characteristics

\begin{tabular}{|c|c|c|c|c|c|c|c|c|}
\hline \multirow{2}{*}{$\begin{array}{l}\text { Characteristics } \\
\text { Age at diagnosis }\end{array}$} & \multicolumn{2}{|c|}{$\begin{array}{l}\text { Total } \\
\mathrm{n}=\mathbf{4 8 1}\end{array}$} & \multicolumn{2}{|c|}{$\begin{array}{l}\text { Training } \\
\text { Cohort } \\
\mathrm{n}=336\end{array}$} & \multicolumn{2}{|c|}{$\begin{array}{l}\text { Validation } \\
\text { Cohort } \\
n=145\end{array}$} & $\chi^{2}$ & $\begin{array}{l}P \\
\text { value* }\end{array}$ \\
\hline & & & & & & & .140 & .929 \\
\hline$<40$ & 50 & $10.40 \%$ & 34 & $10.10 \%$ & 16 & $11.00 \%$ & & \\
\hline $40-64$ & 267 & $55.50 \%$ & 186 & $55.40 \%$ & 81 & $55.90 \%$ & & \\
\hline$\geq 65$ & 164 & $34.10 \%$ & 116 & $34.50 \%$ & 48 & $33.10 \%$ & & \\
\hline Race & & & & & & & .264 & .876 \\
\hline White & 282 & $58.60 \%$ & 198 & $58.90 \%$ & 84 & $57.90 \%$ & & \\
\hline Black & 70 & $14.60 \%$ & 50 & $14.90 \%$ & 20 & $13.80 \%$ & & \\
\hline Others & 129 & $26.80 \%$ & 88 & $26.20 \%$ & 41 & $28.30 \%$ & & \\
\hline Year of diagnosis & & & & & & & .020 & .990 \\
\hline 2000-2005 & 72 & $15.00 \%$ & 50 & $14.90 \%$ & 22 & $15.20 \%$ & & \\
\hline 2006-2011 & 178 & $37.00 \%$ & 125 & $37.20 \%$ & 53 & $36.60 \%$ & & \\
\hline 2012-2016 & 231 & $48.00 \%$ & 161 & $47.90 \%$ & 70 & $48.30 \%$ & & \\
\hline Marital status & & & & & & & 1.006 & .316 \\
\hline Married & 272 & $56.50 \%$ & 185 & $55.10 \%$ & 87 & $60.00 \%$ & & \\
\hline $\begin{array}{l}\text { Unmarried/Loss of } \\
\text { marriage }\end{array}$ & 209 & $43.50 \%$ & 151 & $44.90 \%$ & 58 & $40.00 \%$ & & \\
\hline Grade & & & & & & & 3.863 & .145 \\
\hline I & 23 & $4.80 \%$ & 12 & $3.60 \%$ & 11 & $7.60 \%$ & & \\
\hline II & 227 & $47.20 \%$ & 158 & $47.00 \%$ & 69 & $47.60 \%$ & & \\
\hline III & 231 & $48.00 \%$ & 166 & $49.40 \%$ & 65 & $44.80 \%$ & & \\
\hline AJCC Stage & & & & & & & .557 & .757 \\
\hline - & 55 & $11.40 \%$ & 39 & $11.60 \%$ & 16 & $11.00 \%$ & & \\
\hline II & 164 & $34.10 \%$ & 111 & $33.00 \%$ & 53 & $36.60 \%$ & & \\
\hline III & 262 & $54.50 \%$ & 186 & $55.40 \%$ & 76 & $52.40 \%$ & & \\
\hline ER Status & & & & & & & 3.074 & .080 \\
\hline Positive & 409 & $85.00 \%$ & 292 & $86.90 \%$ & 117 & $80.70 \%$ & & \\
\hline
\end{tabular}




\begin{tabular}{|c|c|c|c|c|c|c|c|c|}
\hline \multirow{2}{*}{$\begin{array}{l}\text { Characteristics } \\
\text { Negative }\end{array}$} & \multicolumn{2}{|c|}{$\begin{array}{l}\text { Total } \\
\mathrm{n}=\mathbf{4 8 1}\end{array}$} & \multicolumn{2}{|c|}{$\begin{array}{l}\text { Training } \\
\text { Cohort } \\
\mathrm{n}=336\end{array}$} & \multicolumn{2}{|c|}{$\begin{array}{l}\text { Validation } \\
\text { Cohort } \\
n=145\end{array}$} & \multirow[t]{2}{*}{$\chi^{2}$} & \multirow[t]{2}{*}{$\begin{array}{l}P \\
\text { value* }\end{array}$} \\
\hline & 72 & $15.00 \%$ & 44 & $13.10 \%$ & 28 & $19.30 \%$ & & \\
\hline \multicolumn{3}{|l|}{ PR Status } & & & & & \multirow[t]{3}{*}{$<.001$} & \multirow[t]{2}{*}{.983} \\
\hline Positive & 348 & $72.30 \%$ & 243 & $72.30 \%$ & 105 & $72.40 \%$ & & \\
\hline Negative & 133 & $27.70 \%$ & 93 & $27.70 \%$ & 40 & $27.60 \%$ & & \\
\hline \multicolumn{3}{|l|}{ HER2 Status】 } & & & & & \multirow[t]{3}{*}{.526} & \multirow[t]{2}{*}{.468} \\
\hline Positive & 75 & $26.10 \%$ & 55 & $27.40 \%$ & 20 & $23.30 \%$ & & \\
\hline Negative & 212 & $73.90 \%$ & 146 & $72.60 \%$ & 66 & $76.70 \%$ & & \\
\hline \multicolumn{3}{|l|}{ Radiotherapy } & & & & & \multirow[t]{3}{*}{.001} & \multirow[t]{2}{*}{.973} \\
\hline Yes & 225 & $46.80 \%$ & 157 & $46.70 \%$ & 68 & $46.90 \%$ & & \\
\hline No & 256 & $53.20 \%$ & 179 & $53.30 \%$ & 77 & $53.10 \%$ & & \\
\hline \multicolumn{3}{|l|}{ Chemotherapy } & & & & & \multirow[t]{3}{*}{1.663} & \multirow[t]{2}{*}{.197} \\
\hline Yes & 349 & $72.60 \%$ & 238 & $70.80 \%$ & 111 & $76.60 \%$ & & \\
\hline No & 132 & $27.40 \%$ & 98 & $29.20 \%$ & 34 & $23.40 \%$ & & \\
\hline \multicolumn{9}{|c|}{$\begin{array}{l}\text { Abbreviation: ER, estrogen receptor; PR, progesterone receptor. HER-2, human epidermal growth facto } \\
\text { receptor } 2 \text {. AJCC, American Joint Committee on Cancer. } \\
\text { * } P \text {-values showed whether there were statistical differences among, new } P \text {-values shall be shown } \\
\text { when necessary in the below. }\end{array}$} \\
\hline \multicolumn{7}{|c|}{$\unrhd$ The SEER database only recorded HER-2 status after January 1, 2010 (317/707). } & & \\
\hline
\end{tabular}

\subsection{Risk Factors for OS in the Training Cohort}

To preliminarily estimate survival outcome, a series of survival analyses were conducted. Among the 481 patients in the current study, a total of $84(17.5 \%)$ patients died during follow-up, and $64(19.4 \%, 64 / 336)$ cases of them were in the training cohort while $20(13.7 \%, 20 / 145)$ cases in the validation cohort, respectively.

Multivariable Cox regression analysis was used to select the prognosis factors affecting OS. In the training cohort, six variables were significantly associated with OS. Adverse prognostic factors included age at diagnosis $(>65, \mathrm{HR}=6.483, P=0.014)$, marital status (unmarried or loss of marriage, $\mathrm{HR}=1.582, P$ $=0.090$ ), AJCC stage ( stage II, HR $=3.750, P=0.048$; stage III, $\mathrm{HR}=9.79, P=0.001$ ), ER negative tumors $(\mathrm{HR}=1.972, P=0.066), \mathrm{PR}$ negative tumors $(\mathrm{HR}=1.837, P=0.063)$, and favorable factor only included chemotherapy (HR $=0.427, P=0.007)$, in terms of OS (Figure 2). 


\subsection{Nomogram Construction}

A nomogram was constructed based on the significantly independent factors identified from the Cox regression model in training cohort (Figure 3). The nomogram demonstrated that AJCC stage had the greatest influence on prediction of OS, followed by age at diagnosis and chemotherapy. ER status, PR status and marital status also revealed moderate influences on OS. The 3- and 5-year survival probabilities were easy to calculate by using the nomogram tool. Take an IMPC female patient diagnosed at the age of 60 as an example, loss of marriage, AJCC stage III, positive ER status and negative PR status, and treatment with BCS and chemotherapy would score a total point of 245.8 , by drawing a downward vertical line we could obtain the probabilities of 3- or 5-year survival for this patient were about $80 \%$ and $65 \%$, respectively.

Figure 3 The prognostic nomogram for 3- and 5-year overall survival (OS).

When using the nomogram model, the upward vertical line drawn for each variable was used to obtain its risk score. Total points were calculated by adding each risk score and drawing a downward vertical line, which illustrated the probability of 3-and 5-year OS.

\subsection{Validation and Calibration of the Nomogram model}

To access the predictive performance of this model, the discrimination, calibration and clinical usefulness were assessed both in the training and validation cohorts. C-index was calculated, and ROC curves were plotted to show discrimination of this nomogram model. The C-indices were $0.771(95 \% \mathrm{Cl}, 0.712$ to $0.830)$ and $0.715(95 \% \mathrm{Cl}, 0.603$ to 0.827$)$ in the training and validation cohorts, respectively. As shown in Figure 4A-D, area under the curve (AUC) values for 3-year and 5-year OS in the training cohort were 0.782 , 0.790 , respectively, and $0.674,0.682$ in the validation cohort, respectively. The calibration curves were applied to assess the calibration, which demonstrated good consistency between the predicted and the observed probability (Figure 4E-H). As shown in Figure 4I, decision curve analysis (DCA) curves illustrated that when the threshold ranged from 0.05 to 0.45 , the net benefit would be positive by using this nomogram, and exhibited a more favorable net benefit for 3- and 5-year OS prediction and a better potential clinical usefulness of this model, when compared to AJCC TNM staging system.

Figure 4 The predictive performance evaluation of this nomogram.

(A-D) The ROC curves of the training cohort (A, B) and the validation cohort (C, D); (E-H) the calibration curves of the training cohort (E, F) and the validation cohort $(G, H)$; (I) The DCA curves for nomogram and AJCC TNM staging of the total cohort. ROC, receiver operator characteristic; DCA, decision curve analysis; AJCC, American Joint Committee on Cancer

\subsection{Kaplan-Meier Survival Analyses and Construction of Risk Score System}


To further validate the predictive ability of this model, we constructed a risk classification through calculation. As shown in Figure 5A and 5B, the OS at 3, 5, and 10 years were $91.60 \%, 84.50 \%$, and $70.00 \%$ in the total cohort, respectively, $92.50 \%, 83.80 \%$, and $66.10 \%$ in the training cohort, respectively, and $89.80 \%, 86.30 \%$, and $79.00 \%$ in the validation cohort, respectively. The $P$ value of 0.140 in Kaplan-Meier survival curves indicated that no significance was found between training and validation cohorts.

The low- and high-risk groups were classified by risk scores, and Kaplan-Meier survival analysis was adopted to compare the two groups in regard to survival-time data for participants in the two risk groups. Risk scores reflecting survival probabilities were calculated using Cox regression analysis for every IMPC patient. According to the median risk score (cutoff $=1.031)$ calculated by the risk score formula mentioned before, the total cohort was separated into the low-risk group $(48.44 \%, 233 / 481)$ and high-risk group $(51.56 \%, 248 / 481)$.

As Figure shown in 5C, the 5-year OS was $72.8 \%$ in the high-risk group, and $96.0 \%$ in the low-risk group. The Kaplan-Meier curves illustrated that participants in the low-risk group had a more favorable OS in comparison with those in the high-risk group $(P<0.001)$, which demonstrated a strong relationship of the risk score with OS. Besides, due to the high rate of ER positive tumors for IMPC patients $(85.00 \%$, 409/481), Kaplan-Meier analysis was performed to evaluate the impact of ER status on prognosis. Figure $5 \mathrm{D}$ indicated that patients with positive ER tumors had a prolonged OS when compared with those with negative ER tumors $(P=0.002)$.

\section{Discussion}

In this research, a predictive model was built based on clinicopathologic features in a large cohort with extended follow-up to predict long-term survival in IMPC patients after BCS. The nomogram model showed a good predictive performance for 3- and 5-year OS after strictly calibrating and validating in both training and validation cohorts. So far as we knew, the current study with large population firstly established a nomogram to provide predictive basis for OS of IMPC patients after BCS. Analyses of demographic variables and clinicopathological factors could provide guidance on the OS and help the decision-making for clinicians and IMPC patients with BCS.

As reported by previous studies[11-14], the objective and reliable prognostic indicators, such as AJCC stage, age at diagnosis, and ER status, have always been seemed as the clinicopathological features which could direct clinical treatment for breast malignancies. In this study, based on the six risk factors affecting OS for IMPC patients undergoing BCS through multivariate Cox regression analyses, the nomogram suggested that AJCC stage contributed most to prognostic prediction, followed by age at diagnosis, ER status and PR status. Similar to previous researches[15-17], it was also found in this study that prognostic markers, such age at diagnosis, stage and hormone receptor status, were long-term survival factors contributing to improved or decreased OS for patients with IMPC. A high tendency of lymph node metastasis was reported in previous IMPC series[18-20], which was closely associated with survival outcome for breast cancer patients[21]. Consequently, it can be inferred that lymph node $(\mathrm{N})$ 
stage utilized by the AJCC has a significant influence on prognosis of IMPC. We also noted that marital status made a moderate contribution to OS, and those who were unmarried or loss of marriage were more at risk in terms of OS. The underlying reasons might be that married patients tended to have more financial resources, chance of surgery and psychosocial support [22], which resulted in a more favorable clinical outcome.

The high tendency of lymphatic vascular invasion, lymph node involvement, as well as a high locoregional recurrence (LRR) risk were supposed to be aggressive clinicopathologic features of IMPC in the previous studies [23-26]. Moreover, OS has always been seemed as an unbiased measurement for survival prediction of breast cancer[11, 12]. In the current research, the 5-year OS for conservatively operated IMPC patients was $80.9 \%$, which was slightly lower than $82.9 \%-90.0 \%$ in recent studies $[13,14$, 26 , 27]. Unexpectedly, the corresponding reasons for the favorable overall survival were still unknown[1, 6 , 20]. Previous studies had identified that large percentage of hormone receptors-positive tumors might contribute to the relatively good prognosis of IMPC $[26,28,29]$. In our study, the ER and PR positivity rates of $85.00 \%$ and $72.30 \%$, respectively, were consistent with the reported literatures that ranged from $20-$ $88 \%[6,30-32]$. In addition, ER-negative tumors were correlated with poor OS (ER negative vs. ER positive: $H R=1.972$ ) for IMPC patients with BCS and contributed moderately to the nomogram model in our study. Survival analyses by risk level in our study showed that ER-positive tumors were associated with a more favorable prognosis. Such findings demonstrated that ER status could be used to predict the prognosis probability for IMPC patients after BCS. The underlying reasons could be attributed to endocrine therapy, which could reduce the potential risk of LRR and improve long-term prognosis[29]. Furthermore, we noted that younger age at diagnosis was a favorable factor for conservatively operated IMPC patients, which was similar to some previous IMPC series[6, 25,29]. On the one hand, the elderly with chronic diseases probably affected overall survival. On the other hand, advances in screening of breast cancer and comprehensive treatment could attribute to the better outcome for young IMPC patients. However, due to lack of prospective study on IMPC of breast, the prediction of long-term survival outcome for IMPC patients after BCS is still required.

According to current guidelines, BCS followed by radiotherapy is one of the standard treatments for invasive breast carcinoma. Correspondingly, IMPC patients were also prone to accept BCS rather than mastectomy in previous series $[5,15,24]$. However, the precise prognosis value of BCS for patients with IMPC remained unknowable. To provide more accurate evidence on prognosis estimation and therapy evaluation in patients with IMPC, a nomogram was constructed based on the significantly independent factors identified from the Cox regression model in training cohort. For instance, if an unmarried woman was diagnosed with stage III and HR positive IMPC when she was 45 years old and had been treated with BCS but without chemotherapy, the OS probabilities at 3- and 5-year were as high as $86.0 \%$ and $70.0 \%$, respectively. Therefore, the nomogram in the present study appeared to be helpful to assess the long-term prognosis, develop therapeutic strategies and improve the compliance of IMPC patients.

Adjuvant/neoadjuvant chemotherapy, which is known to reduce the invasiveness and metastasis of breast cancer, is an essential part of comprehensive treatment for breast malignancies and the mainstay 
of treatment for IMPC patients. After eliminating the confounding factors, the multivariate Cox analyses also revealed chemotherapy as a favorable prognostic factor. Furthermore, in the current nomogram, if the woman patient mentioned above had underwent chemotherapy, the 3-and 5-year cumulative probabilities of survival would be improved to $94.0 \%$ and $87.0 \%$, respectively. Consequently, the administration of chemotherapy could improve the probabilities of OS for IMPC patients and prolong survival time. However, further basic and prospective clinical studies are still warranted.

In addition, predictive performance of the current nomogram was strictly measured on the aspects of discrimination, calibration and clinical usefulness. The C-indices of 0.771 and 0.715 in training and validation cohorts indicated a good discriminatory power of the model and revealed that approximately $75 \%$ of the time this nomogram could help predict the prognosis accurately. In contrast with AJCC staging system, this nomogram showed more favorable clinical usefulness in decision curve analysis and then provided more valuable information for clinical decision making through incorporating more clinicopathological parameters. A risk score system was also established to validate ability of the model to discriminate between patients at different risk levels. If the risk scores of patients were higher than the cut-off of 1.031, the patients would be identified with poorer clinical outcome, and needed further evaluation and more improved comprehensive therapy strategies. Consequently, the current nomogram could provide more accurate prognostic prediction for individual IMPC patients after BCS and be applied to make clinical treatment strategies. Meanwhile, taking account of the complexity of risk score calculation and inconvenient application in clinical practice, the visual nomogram could be performed to calculate the OS probabilities and gave clinicians more accurate assessment of the prognosis for IMPC patients after BCS.

Reflecting on the limitations of this study and the inherent disadvantages of using SEER database[12], some conclusions must be interpreted with caution. Firstly, the design of the study was retrospective and inevitably suffered from possible bias, such as Neyman bias, Berkson bias and lead time bias. Secondly, some essential information was absent in our study and could not be analyzed, such as information on family history, insurance, comorbidities, the percentage of IMPC component, HER-2 status, as well as chemotherapy regimens. Thirdly, records on recurrence and failure patterns were not provided in this database and could not be investigated. Fourthly, statistical significance was defined by $P<0.05$ without adjustment in multiple analysis, consequently the type I error might exceed 0.05 . Moreover, although the nomogram was constructed based on a large population and validated both in the training and validation cohorts, it still needed to be externally validated in other populations.

\section{Conclusion}

In summary, this study found that older age at diagnosis, inexistence of marriage status, late AJCC stage, ER negative or PR negative tumors and not receiving chemotherapy were independent risk factors affecting OS for IMPC patients after BCS. Based on the identified variables to provide prognostic prediction for conservatively operated IMPC patients, we developed and validated a nomogram that could accurately predict the individualized risk of OS for patients with IMPC after BCS. By identifying the risk 
stratification, this model was expected to offer guidance for making treatment decision in improving longterm follow-up strategies. Randomized controlled clinical trials with long follow-up time are still needed to provide a high level of evidence on the predictive probabilities of OS for conservatively operated IMPC patients.

\section{Declarations}

\section{Funding}

This study was supported by the National Natural Science Foundation of China (NSFC 81502413 to C. Zhou) and Shaan'xi Provincial Natural Science Foundation of China (SNSFC 2019SF-145 to C. Zhou).

\section{Conflict of interest}

The authors declare that there is no interest of conflict.

\section{Author Contributions}

YZ drafted the manuscript and analyze data; SL, LY and ZY generated the figure; NC and PQ performed the background research; JZ and $\mathrm{HZ}$ carried out additional analyses; $\mathrm{CZ}$ and $\mathrm{JH}$ edited the manuscript. All authors have read and approved the content of the manuscript.

\section{Acknowledgments}

We are thankful for the Surveillance, Epidemiology, and End Results Program (National Cancer Institute) for development of the SEER database. We wish to thank all our colleagues in the Departments of Breast Surgery, First Affiliate Hospital of Xi'an Jiaotong University

\section{Ethical statement}

This study was approved by the Ethical Committee of the First Affiliated Hospital of Xi'an Jiaotong University. The SEER data erases the identity information of patients, so there is no need for informed consent from the patients.

\section{References}

1. Gokce H, Durak MG, Akin MM, Canda T, Balci P, Ellidokuz H et al: Invasive micropapillary carcinoma of the breast: a clinicopathologic study of 103 cases of an unusual and highly aggressive variant of breast carcinoma. Breast J 2013, 19(4):374-381.

2. Ide $\mathrm{Y}$, Horii R, Osako T, Ogura $\mathrm{K}$, Yoshida R, Iwase $T$ et al: Clinicopathological significance of invasive micropapillary carcinoma component in invasive breast carcinoma. Pathol Int 2011, 61(12):731736. 
3. Nassar H, Wallis T, Andea A, Dey J, Adsay V, Visscher D: Clinicopathologic analysis of invasive micropapillary differentiation in breast carcinoma. Mod Pathol 2001, 14(9):836-841.

4. Jones KN, Guimaraes LS, Reynolds CA, Ghosh K, Degnim AC, Glazebrook KN: Invasive micropapillary carcinoma of the breast: imaging features with clinical and pathologic correlation. AJR Am J Roentgenol 2013, 200(3):689-695.

5. Vingiani A, Maisonneuve P, Dell'orto P, Farante G, Rotmensz N, Lissidini G et al: The clinical relevance of micropapillary carcinoma of the breast: a case-control study. Histopathology 2013, 63(2):217224.

6. Chen H, Wu K, Wang M, Wang F, Zhang M, Zhang P: Invasive micropapillary carcinoma of the breast has a better long-term survival than invasive ductal carcinoma of the breast in spite of its aggressive clinical presentations: a comparison based on large population database and case-control analysis. Cancer Med 2017, 6(12):2775-2786.

7. Kuroda H, Sakamoto G, Ohnisi K, Itoyama S: Clinical and pathologic features of invasive micropapillary carcinoma. Breast Cancer 2004, 11(2):169-174.

8. Veronesi U, Cascinelli N, Mariani L, Greco M, Saccozzi R, Luini A et al: Twenty-year follow-up of a randomized study comparing breast-conserving surgery with radical mastectomy for early breast cancer. N Engl J Med 2002, 347(16):1227-1232.

9. Fisher B, Anderson S, Bryant J, Margolese RG, Deutsch M, Fisher ER et al: Twenty-year follow-up of a randomized trial comparing total mastectomy, lumpectomy, and lumpectomy plus irradiation for the treatment of invasive breast cancer. N Engl J Med 2002, 347(16):1233-1241.

10. Terry M. Therneau PM: Modeling Survival Data: Extending the Cox Model: Springer; 2000.

11. Ma Y, Yang Z, Gao Y, Li K, Qiu P, Chen H et al: Research on the Role of Combined Chemotherapy and Radiotherapy in Patients With N+ Non-Metastatic Metaplastic Breast Carcinoma: A Competing Risk Analysis Model Based on the SEER database, 2000 to 2015. Frontiers in oncology 2020, 10:583488.

12. Li Y, Zhang H, Zhang W, Ren Y, Qiao Y, Li K et al: A Competing Risk Analysis Model to Determine the Prognostic Value of Isolated Tumor Cells in Axillary Lymph Nodes for T1NOMO Breast Cancer Patients Based on the Surveillance, Epidemiology, and End Results Database. Frontiers in oncology 2020, 10:572316.

13. Li W, Han Y, Wang C, Guo X, Shen B, Liu F et al: Precise pathologic diagnosis and individualized treatment improve the outcomes of invasive micropapillary carcinoma of the breast: a 12-year prospective clinical study. Mod Pathol 2018, 31(6):956-964.

14. Chen AC, Paulino AC, Schwartz MR, Rodriguez AA, Bass BL, Chang JC et al: Prognostic markers for invasive micropapillary carcinoma of the breast: a population-based analysis. Clin Breast Cancer 2013, 13(2):133-139.

15. Li D, Zhong C, Cheng Y, Zhu N, Tan Y, Zhu L et al: A Competing Nomogram to Predict Survival Outcomes in Invasive Micropapillary Breast Cancer. J Cancer 2019, 10(27):6801-6812.

16. Meng X, Ma H, Yin H, Yin H, Yu L, Liu L et al: Nomogram Predicting the Risk of Locoregional Recurrence After Mastectomy for Invasive Micropapillary Carcinoma of the Breast. Clinical breast 
cancer 2020.

17. Ye F-G, Xia C, Ma D, Lin P-Y, Hu X, Shao Z-M: Nomogram for predicting preoperative lymph node involvement in patients with invasive micropapillary carcinoma of breast: a SEER population-based study. BMC cancer 2018, 18(1):1085.

18. Ye F, Yu P, Li N, Yang A, Xie X, Tang H et al: Prognosis of invasive micropapillary carcinoma compared with invasive ductal carcinoma in breast: A meta-analysis of PSM studies. Breast 2020, 51:11-20.

19. Walsh MM, Bleiweiss IJ: Invasive micropapillary carcinoma of the breast: eighty cases of an underrecognized entity. Hum Pathol 2001, 32(6):583-589.

20. Yu JI, Choi DH, Huh SJ, Cho EY, Kim K, Chie EK et al: Differences in Prognostic Factors and Failure Patterns Between Invasive Micropapillary Carcinoma and Carcinoma With Micropapillary Component Versus Invasive Ductal Carcinoma of the Breast: Retrospective Multicenter Case-Control Study (KROG 13-06). Clin Breast Cancer 2015, 15(5):353-361.e351-352.

21. Soerjomataram I, Louwman MWJ, Ribot JG, Roukema JA, Coebergh JWW: An overview of prognostic factors for long-term survivors of breast cancer. Breast cancer research and treatment 2008, 107(3):309-330.

22. Zhai Z, Zhang F, Zheng Y, Zhou L, Tian T, Lin S et al: Effects of marital status on breast cancer survival by age, race, and hormone receptor status: A population-based Study. Cancer medicine 2019, 8(10):4906-4917.

23. Wu Y, Zhang N, Yang Q: The prognosis of invasive micropapillary carcinoma compared with invasive ductal carcinoma in the breast: a meta-analysis. BMC Cancer 2017, 17(1):839.

24. Yoon GY, Cha JH, Kim HH, Shin HJ, Chae EY, Choi WJ: Comparison of invasive micropapillary and invasive ductal carcinoma of the breast: a matched cohort study. Acta Radiol 2019, 60(11):14051413.

25. Hao S, Zhao YY, Peng JJ, Ren F, Yang WT, Yu KD et al: Invasive micropapillary carcinoma of the breast had no difference in prognosis compared with invasive ductal carcinoma: a propensitymatched analysis. Sci Rep 2019, 9(1):286.

26. Chen AC, Paulino AC, Schwartz MR, Rodriguez AA, Bass BL, Chang JC et al: Population-based comparison of prognostic factors in invasive micropapillary and invasive ductal carcinoma of the breast. Br J Cancer 2014, 111(3):619-622.

27. Lewis GD, Xing Y, Haque W, Patel T, Schwartz M, Chen A et al: Prognosis of lymphotropic invasive micropapillary breast carcinoma analyzed by using data from the National Cancer Database. Cancer Commun (Lond) 2019, 39(1):60.

28. Luna-More S, Casquero S, Perez-Mellado A, Rius F, Weill B, Gornemann I: Importance of estrogen receptors for the behavior of invasive micropapillary carcinoma of the breast. Review of 68 cases with follow-up of 54. Pathol Res Pract 2000, 196(1):35-39.

29. Wu SG, Zhang WW, Sun JY, Li FY, Chen YX, He ZY: Postoperative radiotherapy for invasive micropapillary carcinoma of the breast: an analysis of Surveillance, Epidemiology, and End Results 
database. Cancer Manag Res 2017, 9:453-459.

30. Yang YL, Liu BB, Zhang X, Fu L: Invasive Micropapillary Carcinoma of the Breast: An Update. Arch Pathol Lab Med 2016, 140(8):799-805.

31. Badyal RK, Bal A, Das A, Singh G: Invasive Micropapillary Carcinoma of the Breast: Immunophenotypic Analysis and Role of Cell Adhesion Molecules (CD44 and E-Cadherin) in Nodal Metastasis. Appl Immunohistochem Mol Morphol 2016, 24(3):151-158.

32. Pettinato G, Manivel CJ, Panico L, Sparano L, Petrella G: Invasive micropapillary carcinoma of the breast: clinicopathologic study of 62 cases of a poorly recognized variant with highly aggressive behavior. American journal of clinical pathology 2004, 121(6):857-866.

\section{Figures}

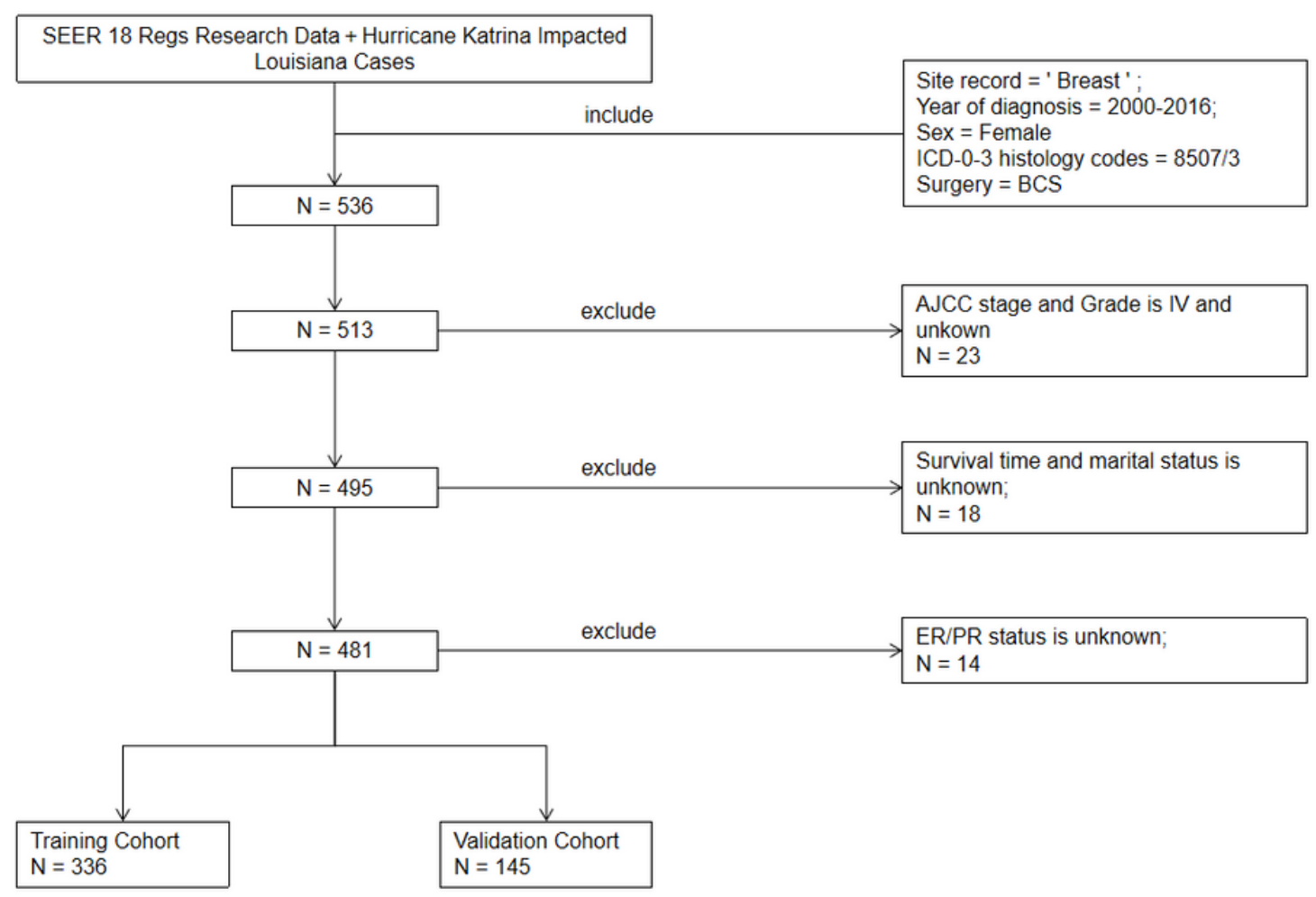

\section{Figure 1}

Eligibility, inclusion, and exclusion criteria of study population 


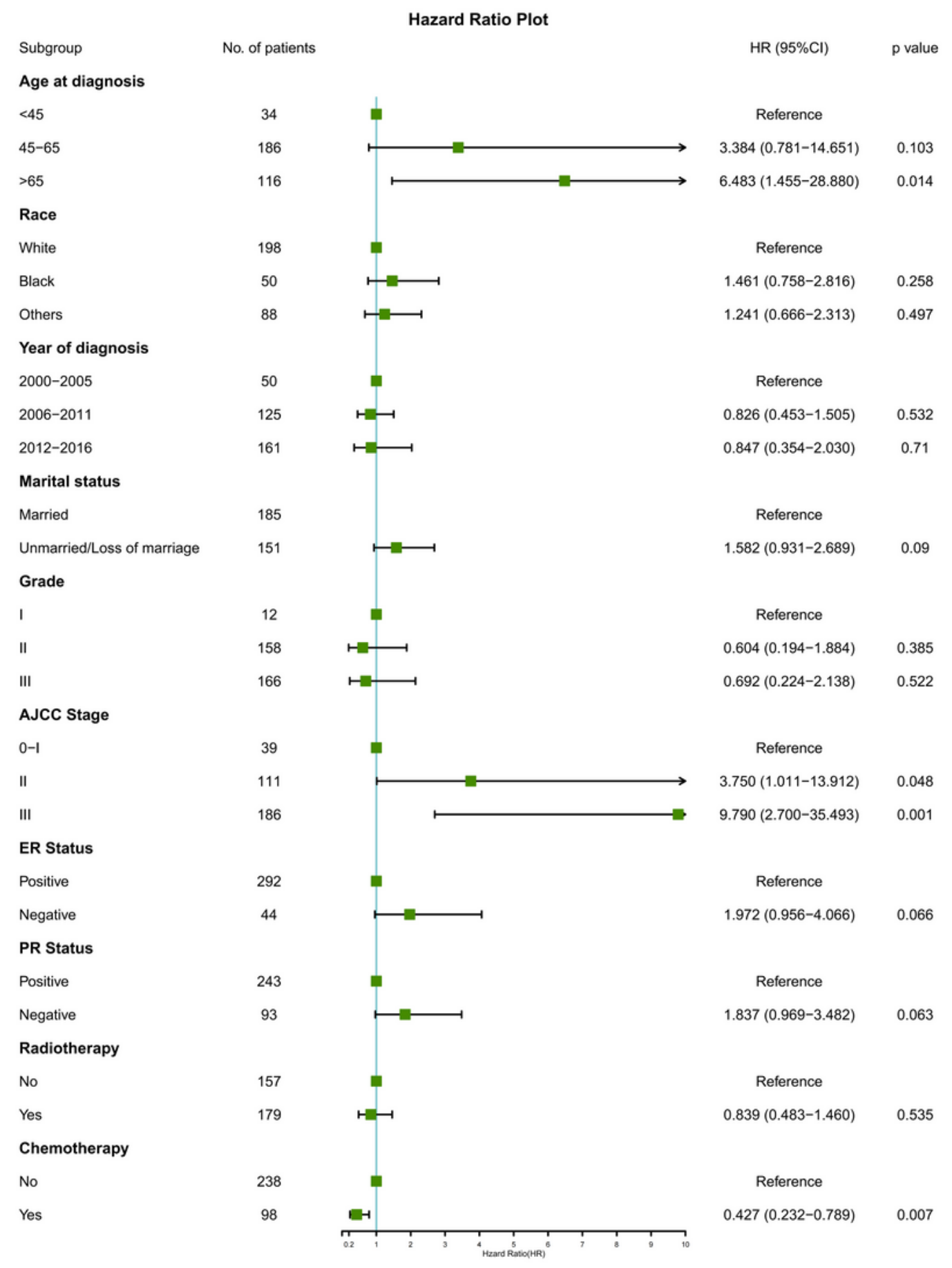

\section{Figure 2}

The forest plot for multivariate Cox analysis in the training cohort 


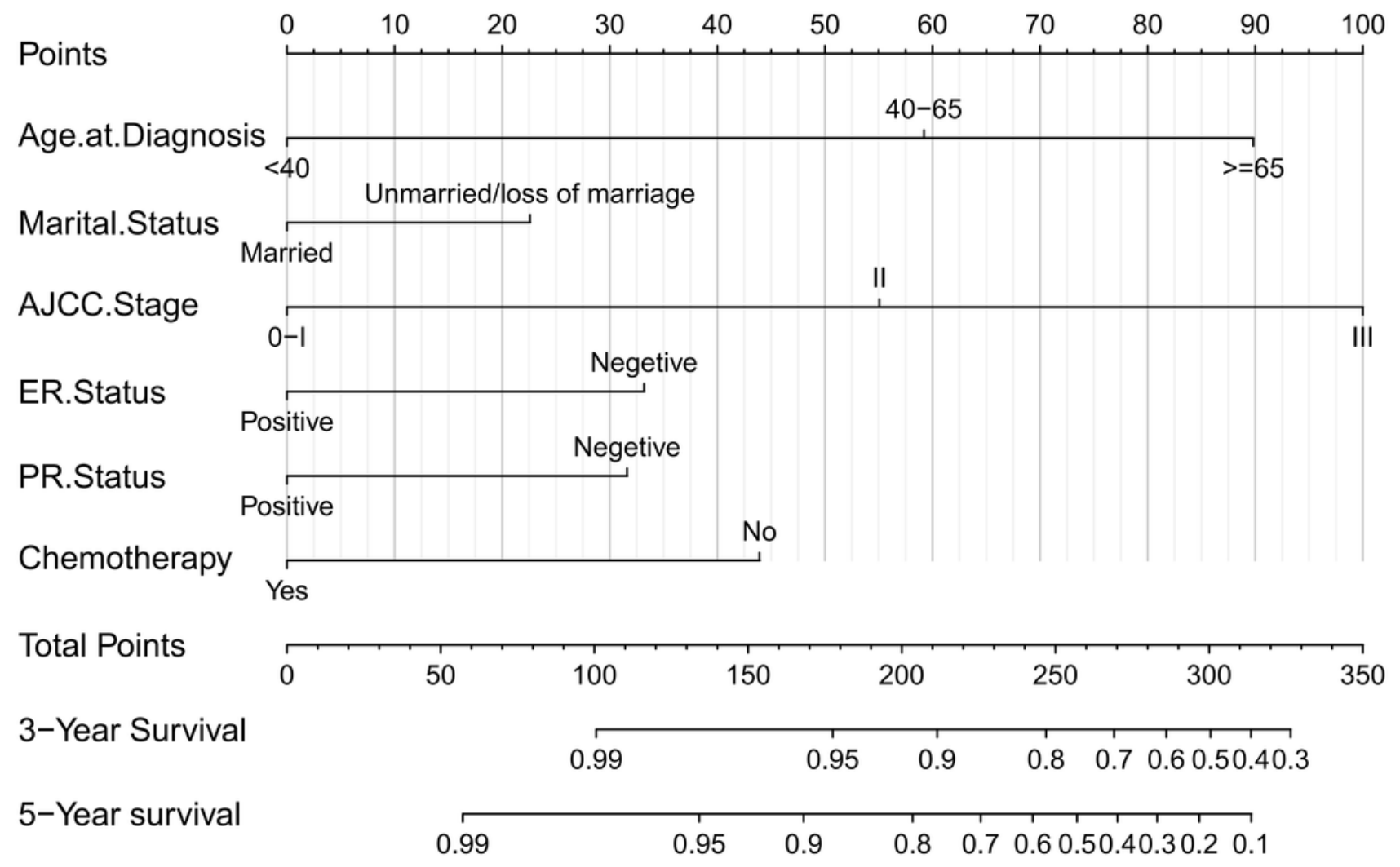

\section{Figure 3}

The prognostic nomogram for 3- and 5-year overall survival (OS). 

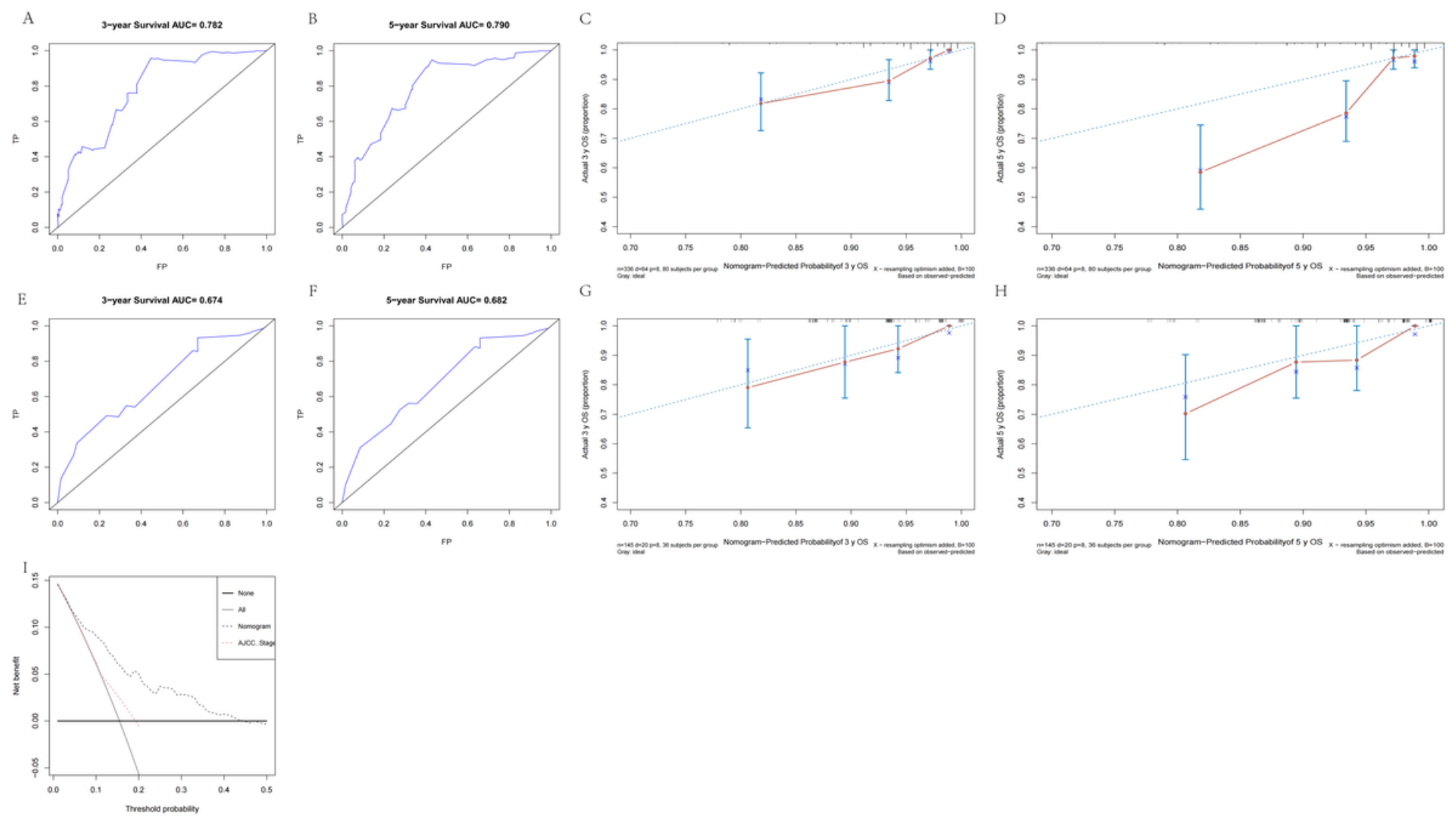

\section{Figure 4}

The predictive performance evaluation of this nomogram.

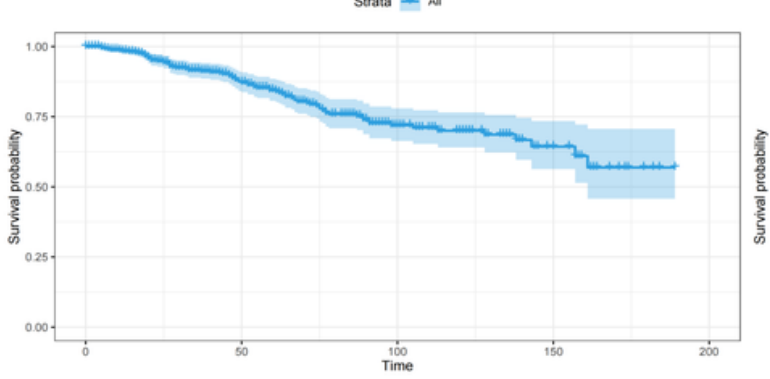

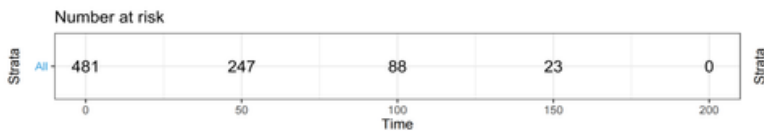

C

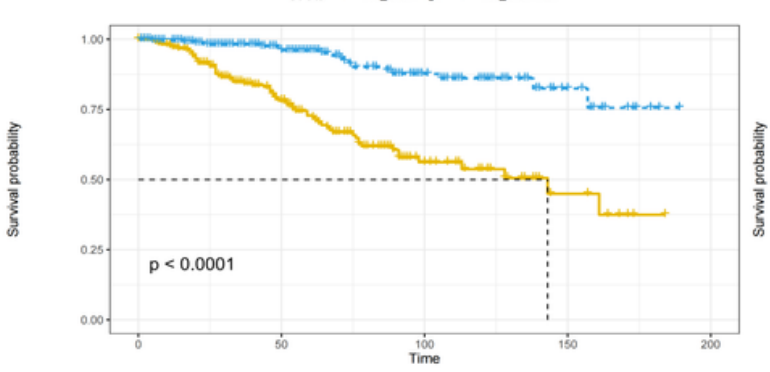

B
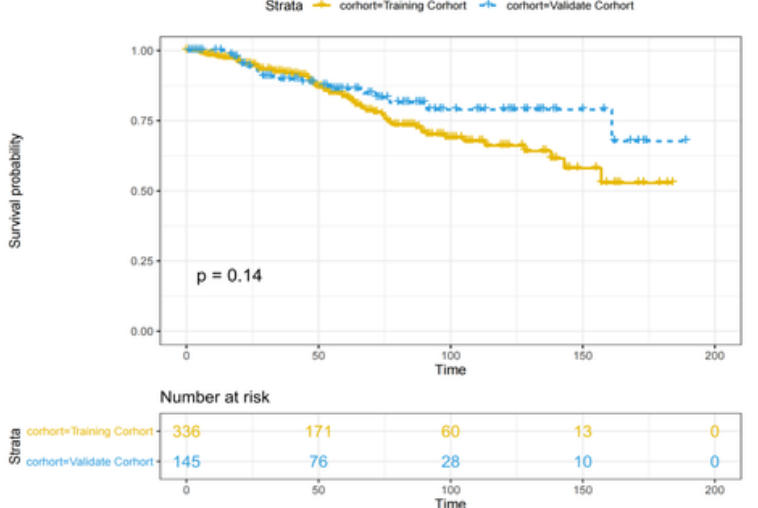

D

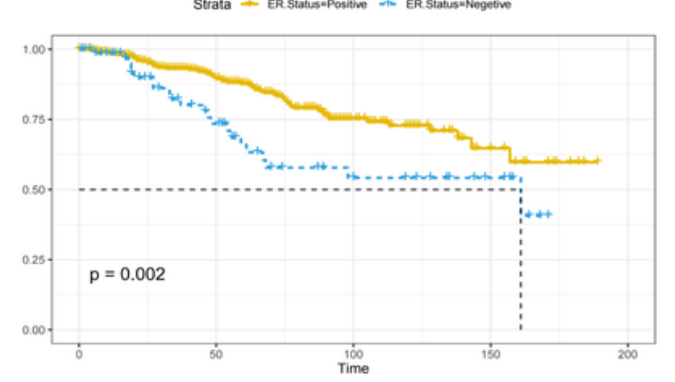

Number at risk

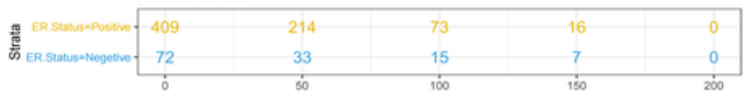




\section{Figure 5}

Survival analysis (A) in the total cohort. Subgroup analyses stratified (B) by cohort; (C) by risk score; (D) ER (estrogen receptor). P-value was calculated by log-rank test 